\title{
A Spectral Filter Analyzer for Sampling of Optical Bragg Grating Sensors
}

\author{
N. Samfirescu*, Th. Tschudi", M. A. Bader*, K. Plies**, G. Jakob*, Th. Schanze ${ }^{* *}$ \\ ${ }^{*}$ Photonik Zentrum Hessen in Wetzlar AG, ${ }^{* *}$ CORRSYS 3D Sensors AG \\ Charlotte-Bamberg-Str. 6 \\ D-35578 Wetzlar
}

\begin{abstract}
In order to measure a Bragg grating sensor, a new combination of optical and electronical components has been developed for light intensity independent conversions of wavelength into electrical signals. The spectral analyzer allows fast, stable, and precise measurements of strain and temperature with optical Bragg grating sensors.
\end{abstract}

\section{Introduction}

In order to sample optical sensors based on Bragg gratings, different types of interrogators are currently in use: interferometers, tunable lasers and devices employing spectral filters for wavelength-amplitude conversions. Most of the commercially available interrogators are very expensive. However, for many applications including civil structure monitoring (Figure 1) economic optical C-band interrogators are desired.
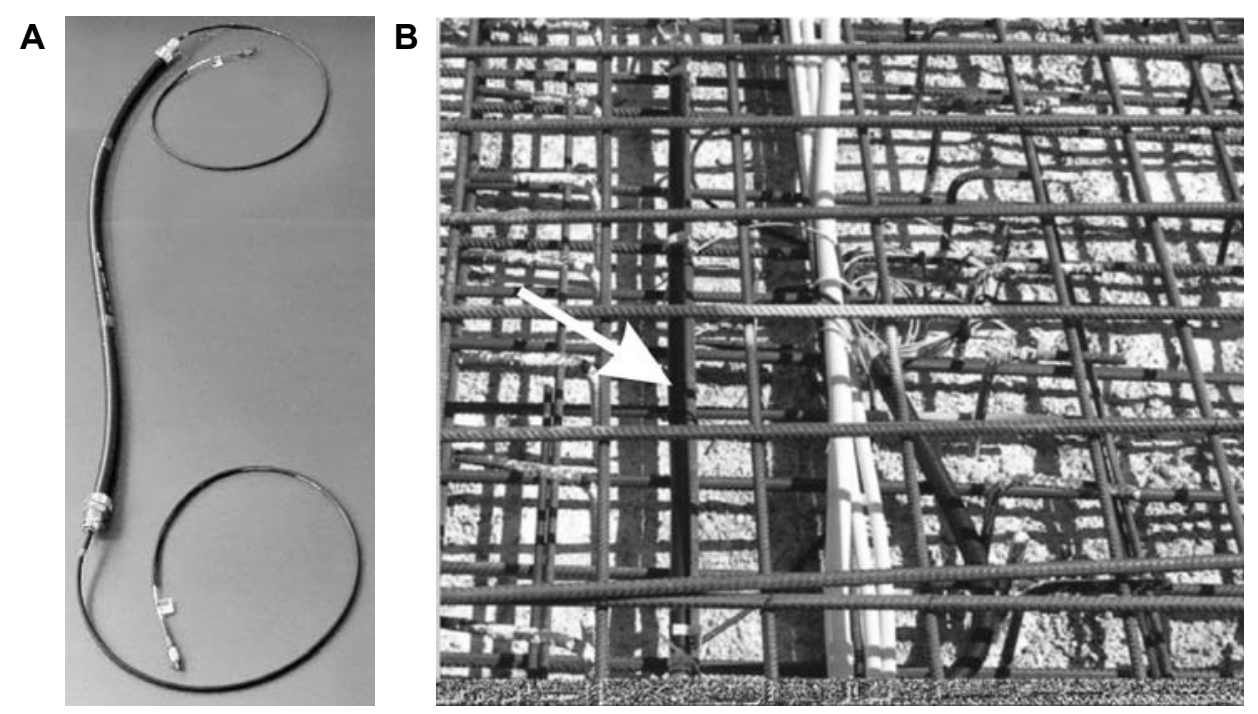

Figure 1. A. Fiber Bragg sensor designed for civil structure monitoring. B. AOS-fibre Bragg sensor embedded in a concrete bridge: "Hausertorbrücke", Wetzlar; white arrow.

\section{Method}

To measure a fibre Bragg grating response a small spectral sampling bandwidth is very often sufficient, e.g. $20 \mathrm{~nm}$ (Figure 2). In order to obtain an intensity independent conversion of light at a given Bragg 
wavelength $\lambda_{\text {Bragg }}$ into an electrical signal adequate for subsequent signal processing a new combination of optical and electronical components has been developed (Figure 3). An sufficiently bright infrared light source, superluminescent light emitting diode (SLED), is connected by optical fibres to a coupler which also directs the reflected light from the fibre Bragg grating (FBG) to an opto-electronical detector.

The detection principle consists of a beam splitter, two collimator lenses and two spectral filters (Figure 3). The spectral transfere functions of the filters were selected to be monotonic and, to increase sensitivity and signal-to-noise ratio, complementary (Figure 4). The transmitted light of a filter is subsequently converted by a photodiode to an electrical signal, i.e. an electrical current $I$ that is related to $\lambda: I=I(\lambda)$. Note that this relationsship has to be monotone. According to the filter transfere functions we find: $\lambda_{\text {Bragg }} \uparrow \downarrow \Rightarrow I_{1} \downarrow \uparrow$ and $I_{2} \uparrow \downarrow$. The contrast of the two photodiode currents $S=\left(I_{1}-I_{2}\right) /\left(I_{1}+I_{2}\right)$ is, as required, a monotonic function of the wavelength $\lambda_{\text {Bragg }}$. Due to our chip integrated photodiode approach, the obtained contrast signal is highly compensated against fluctuations of the light source and aging or temperature drift. However, the signal quality depends significantly on the filter quality and not on other the detector properties which prospects a cost-effective system.
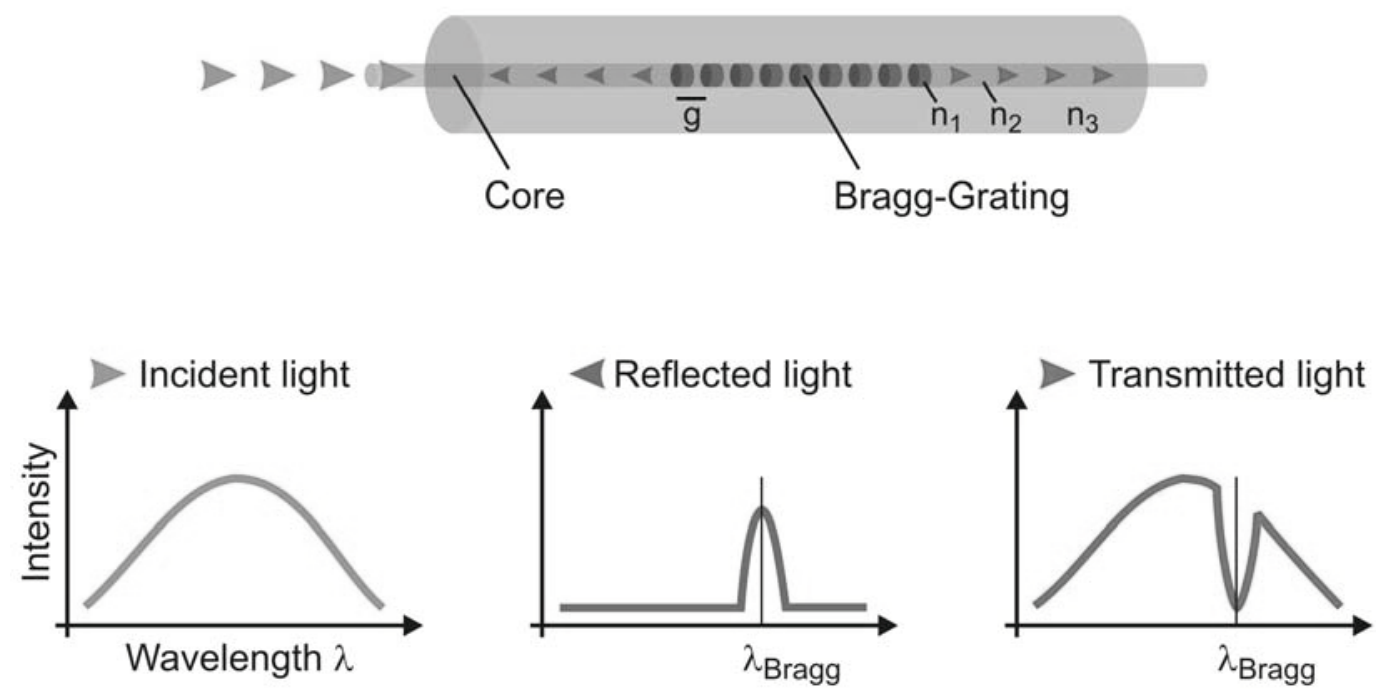

B

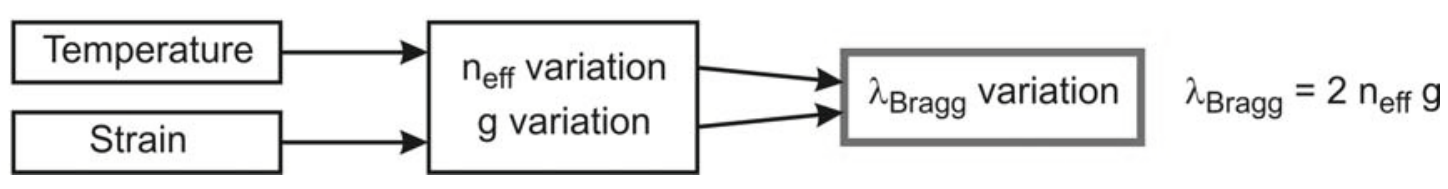

Figure 2. Principle of a fibre Bragg grating. A fiber Bragg grating (FBG) is a type of distributed Bragg reflector constructed in a short segment of an optical fiber. An FBG reflects light of a particular wavelength and transmits light with other wavelengths. The particular wavelength, called Bragg wavelength, is related to grating's strain and temperature: $\lambda_{\text {Bragg }}=\lambda_{\text {Bragg }}(\varepsilon, T)=2 n_{\text {eff }}(\varepsilon, T) g(\varepsilon, T)$. For many FBG $\lambda_{\text {Bragg }}$ is a monotonic function of strain $\varepsilon$ and temperature $T$. 


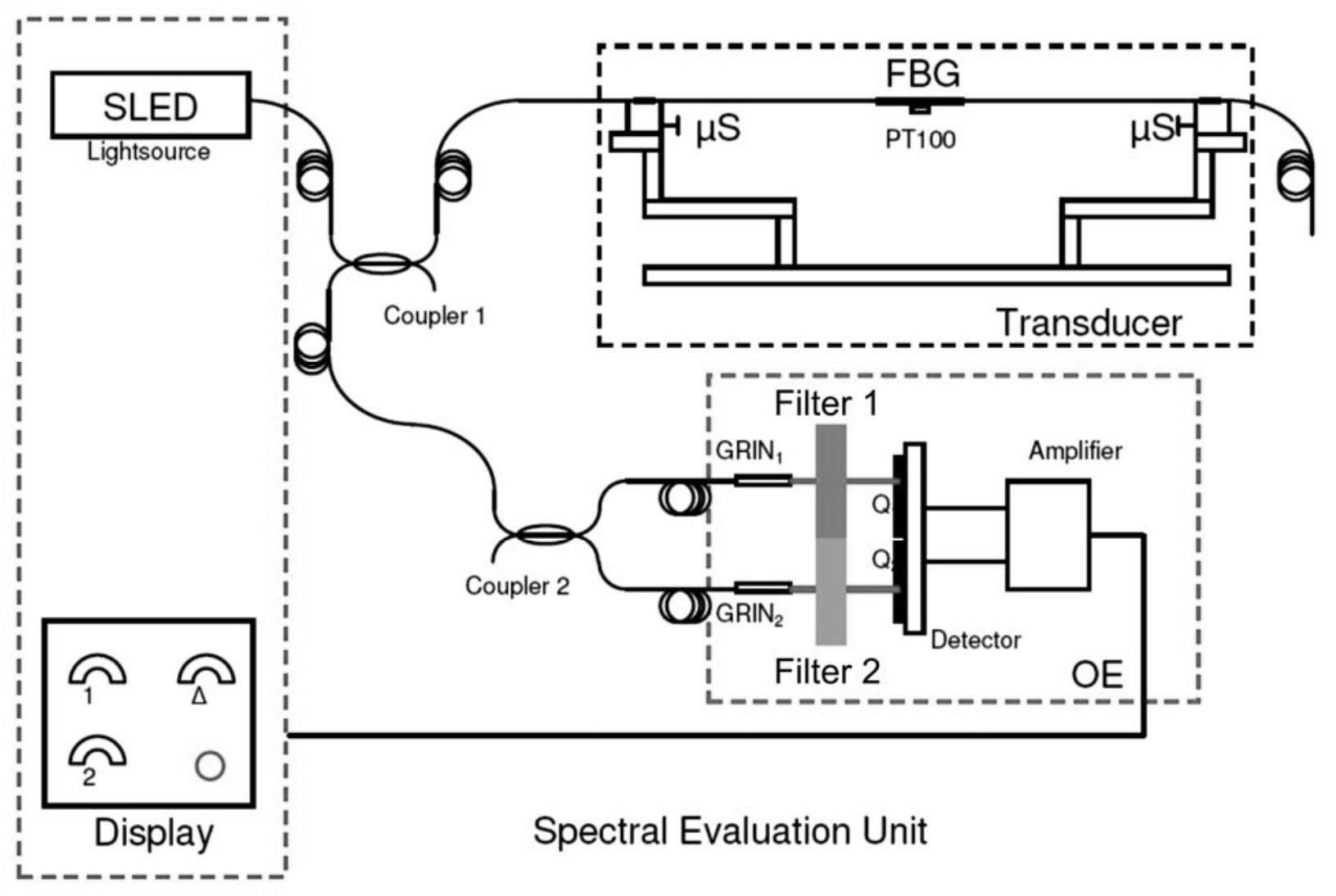

Figure 3. Scheme of our approach. The light of a SLED ist transmitted to a FBG. The reflected FBG light is projected onto two filters with well defined transfer functions (see Figure 4) and subsequently converted by photodiode detectors to electrical currents. The currents are amplified and subsequently processed: analogue-to-digital conversion and digital signal processing (not shown).

\section{Filter transfer functions}

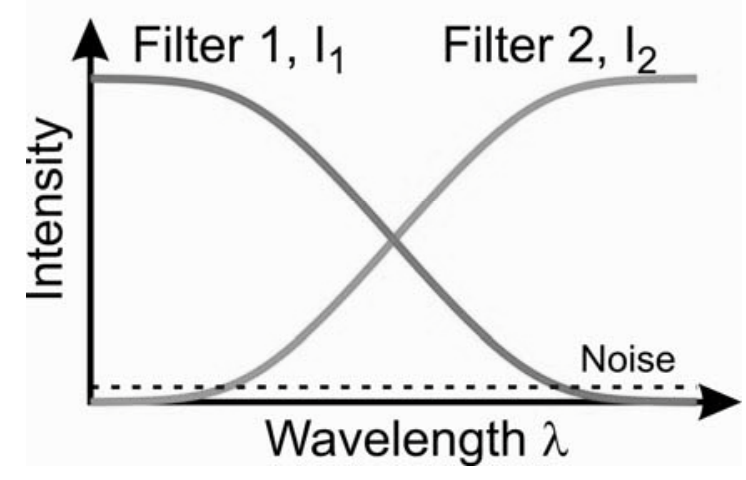

Figure 4. Transfer functions of the spectral filters. Using such filters the current contrast of the photodiodes $S=\left(I_{1}-I_{2}\right) /\left(I_{1}+I_{2}\right)$ is a monotonic function of $\lambda$ and thus invariant to light intensity changes (see Figure 3). 


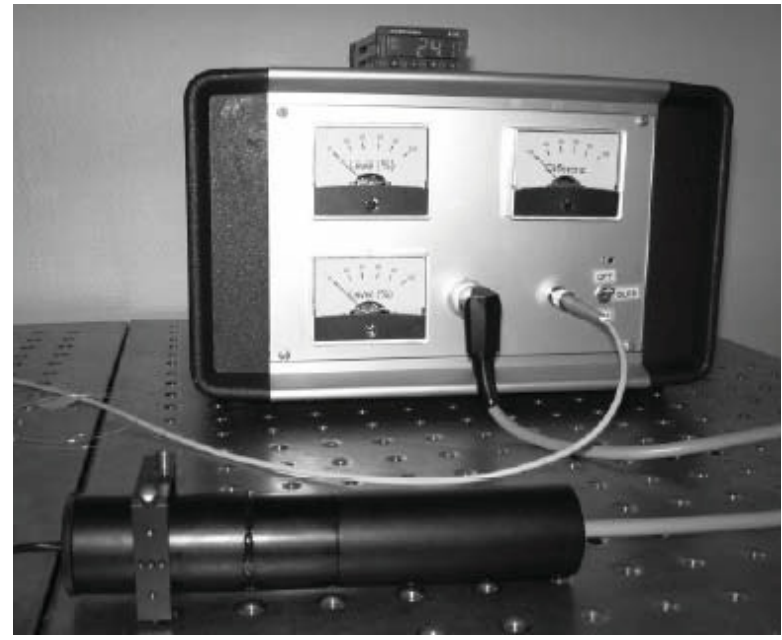

Figure 5. The prototype.

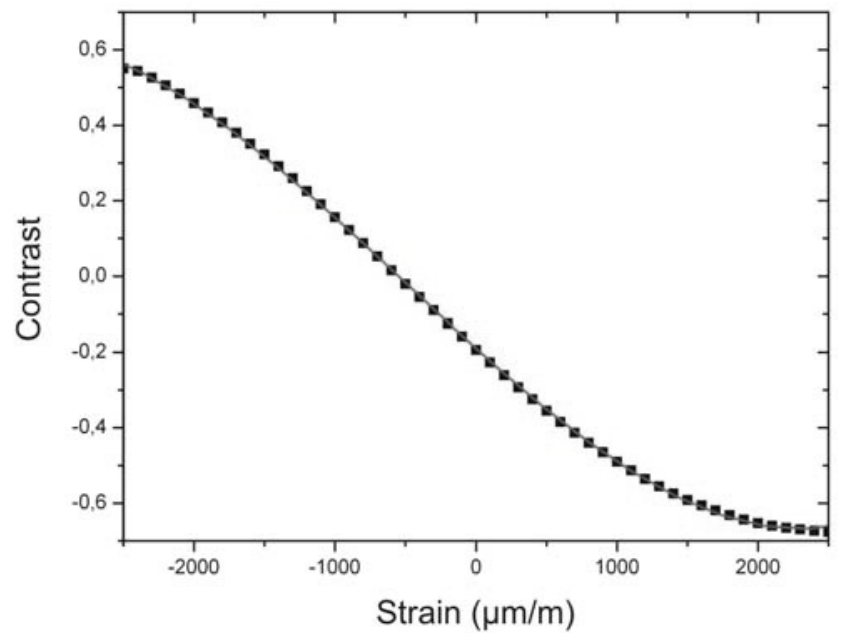

Figure 6. Contrast-strain operating characteristic curve computed by $S=\left(I_{1}-I_{2}\right) /\left(I_{1}+I_{2}\right)$, where $I_{1}$ and $I_{2}$ are photodiode currents.

\section{Results}

In order to demonstrate the feasibility of our approach a first prototype of a C-band analyzer/interrogator using spectral filters has been developed. (Figure 5). The operating range of the system is about $10 \mathrm{~nm}$, thus adapted to C-band or $1500 \mathrm{~nm}$ fibre Bragg gratings. The spectral resolution of the system is less than $25 \mathrm{pm}$ in a spectral operating range of about $5 \mathrm{~nm}$. Potential interference patterns due to the spectral filters were prevented by a special treatment of the filter surfaces. The achieved linearity and stability of the system depends very strongly on the characteristics of the spectral filters and not on the photodiodes or other electronical components.

An example of a strain measurement with a Bragg sensor and this new spectral analyzer is shown in Figure 6. Using high quality filters the results of our approach are - to our knowledge - in good correspondence with other principles for Bragg grating sensors designed for strain or temperature measurements.

Currently we are working towards a flexible multi-channel system for cost-effective civil structure monitoring.

\section{Discussion and Conclusions}

We have developed a new spectral analyzer by employing two spectral filters for wavelength-amplitude conversion for each channel. The filter transfer functions were designed to optimize sensitivity and signalto-noise ratio. A light intensity independent spectral analysis was obtained by contrast function computation. The manufactured spectral analyzer prototype allows fast, stable and precise measurements of strain and temperature with optical Bragg grating sensors. Due to its design our spectral analyzer is insensitive to aging or temperature drift of the detector unit and it is well suited for cost effective applications. 


\section{Acknowledgements}

The authors gratefully acknowledge the support by Hessen Agentur $\mathrm{GmbH}$, Wiesbaden, Germany, and EU (EFRE): Hessen Agentur project 95/06-01 and "dieses Projekt (HA-Projekt-Nr.: 164/08-26) wird im Rahmen der Innovationsförderung des Landes Hessen gefördert, finanziert aus Mitteln der LOEWE Landes-Offensive zur Entwicklung Wissenschaftlich-ökonomischer Exzellenz, Förderlinie 3: KMUVerbundvorhaben“.

Picture credits (@). Fig. 1A: AOS GmbH, Dresden, Germany, 2006. Figs. 1B, 2: CORRSYS 3D Sensors AG, Wetzlar, Germany, 2006/7. Fig. 3: Photonikzentrum Hessen in Wetzlar AG, Wetzlar, Germany, 2007. Fig. 4: CORRSYS 3D Sensors AG, Wetzlar, Germany. Figs. 5, 6: Photonikzentrum Hessen in Wetzlar AG, Wetzlar, Germany, 2008.

\section{References}

Dakin J, Dakin C (1997) Optical Fiber Sensors - Volume Four; Applications, Analysis, and Future Trends. Artech House, Boston London

López-Higuera JM (2002) Handbook of Optical Fibre Technology. John Wiley \& Sons

Othonos A (1997) Fiber Bragg gratings. Rev Sci Instrum 68, 4309-4340

Othonos A, Kalli K (1999) Fiber Bragg Gratings: Fundamentals and Applications in Telecommunications and Sensing. Artech House Inc.

Samfirescu N (2007) The Development of a spectral evaluation unit for strain measurements", diploma thesis, Physics Faculty, University of Siegen

Samfirescu N, Bader MA, Schanze T (2008) A novel spectral filter analyzer for Bragg grating based strain and temperature measurements". Workshop: Optical Spectrometer: Design, Technology, Application and Trend, IPHT Jena $\left(11^{\text {th }}-12^{\text {th }}\right.$ March 2008)

Tschudi T, Blin R, Schmidt M (2007) Optical spectrometer. Patent WO002007082678A1

Yu FTS, Yin S (2002) Fiber Optic Sensors. Marcel Dekker Inc., New York 